\title{
La tarea docente en el modo de actuación profesional pedagógico de estudiantes de Biología-Química
}

\section{The teaching task in the pedagogical performance in students of Biology And Chemestry}

Alba María Lopetegui Canel'alba.lopetegui@upr.edu.cu

\section{Resumen}

El proceso de formación del modo de actuación profesional pedagógico es parte esencial de la formación de los profesionales de la educación. Este trabajo tiene como objetivo elaborar tareas docentes centradas en la determinación y solución de problemas didácticos, de forma tal que propicie el desarrollo del modo de actuación profesional pedagógicodurante el proceso enseñanza-aprendizajede la formación inicial de los profesores de Biología y Química en la Universidad de Pinar del Río "Hermanos Saíz Montes de Oca".A partir de los resultados de un diagnóstico del modo de actuación profesional pedagógico de los estudiantes de la carrera Biología-Química, se identificaron problemas didácticos a solucionar en el proceso de enseñanza-aprendizaje de la carrera Biología-Química. Con la solución de estos problemas didácticos se contribuye a desarrollarun modo de actuación profesional pedagógico.Como métodos teóricos se emplearon el histórico y el lógico, el análisis y la síntesis, la inducción y la deducción, el hipotético-deductivo y el enfoque sistémico-estructural. Como métodos empíricos la encuesta y el pre-experimento. Como método estadístico-matemático se utilizó el cálculo porcentual. Con la aplicación del pre-experimento se pudo constatar en la práctica pedagógica la validez de la propuesta de las tareas docentes elaboradas.

Palabras claves: Modo de actuación profesional pedagógico.

\section{Abstract}

The process of formation of the pedagogical professional behavior is an essential part of the formation of professionals of education. This research targets the objective of elaborating professional tasks focused on the determination and solution of didactic problems, so as to develop the pedagogical professional behavior during the teachinglearning process of the initial formation of Biology and Chemistry teachers at "HermanosSaiz Montes de Oca" University in Pinar del Río. Starting from the diagnosis of the pedagogical professional behavior of the students, from such careers, it was determined the didactic problems to solve in the teaching and learning processes. With the solution of these didactic problems, there is a contribution to the development of the pedagogical professional behavior. As theoretical methods, there were used: the historical logical, analysis- synthesis, induction and deduction, hypothetical- deductive methods and the systemic-structural approach. As empirical methods, there were used: the survey and the pre-experiment. As statistical-mathematical method, there was used the percentage calculation. With the application of the pre experiment, it was assessed the validity of the proposal of the professional tasks elaborated in the pedagogical practice.

\footnotetext{
${ }^{1}$ Máster en Ciencias. Profesor Auxiliar. Universidad de Pinar del Río. Cuba
} 
Key words: pedagogical professional performance.

\section{Introducción}

Con la presentación de este trabajo es posible analizar algunas de las experiencias alcanzadas como resltados de investigación en los procesos de formación de los profesionales de la educación desde la Universidad partiendo desde las tendencias de desarrollo y perspectivas de la Educación Superior. Se presentan resultados efectivos en el trabajo de la Universidad en la construcción de un mundo mejor.

En la formación de profesionales de la educación existen antecedentes inmediatos tanto en el ámbito internacional y nacional en la última década, los que evidencian un proceso continuo de transformaciones de las concepciones existentes acerca del profesorado y su formación desde y para la práctica, en los diferentes contextos de actuación profesional.

Ese proceso ha estado acompañado, en Cuba, de estudios acerca del modo de actuación profesional realizado por muchos investigadores entre los cuales se destacaÁlvarez (1999),quien aborda la temática desde una perspectiva muy amplia, al hacerlo para la formación de profesionales en general.

En tanto, García (1997)Chirino (2002) yFuxá (2004) refieren que el núcleo del perfeccionamiento del modo de actuación profesional pedagógico es la auto preparación del maestro; Addine(2004) lo define según sus atributos principales; Calzado (2003) precisa el tratamiento al modo de actuación profesional pedagógico desde la Didáctica en la organización del proceso de enseñanza-aprendizaje. Pla (2005), centra su atención en la dirección del proceso pedagógico en general, basado en una secuencia de acciones generales.

Otra investigadora que ha realizado sus aportes respecto al tema esPáez (2010), quien hace el estudio del modo de actuación profesional pedagógico interdisciplinario, en los estudiantes en formación como docentes, para la Secundaria Básica.

En el actual contexto histórico marcado por un vertiginoso desarrollo científico- técnico la formación de los profesionales de la educación tiene una gran importancia, como ya se ha expresado en párrafos anteriores, en especial la formación de los profesores de las Ciencias Naturales y en particular los de Biología y Química en las Universidades, los que deben ser capaces de lograr la formación integral de las nuevas generaciones materializando la dirección del proceso de enseñanza-aprendizaje de las asignaturas de las que son especialistas.

Al realizar el diagnóstico a estudiantes de la carrera Biología-Química se determina en ellos un accionar limitado relacionado con la búsqueda de nuevos conocimientos a partir de la integración de contenidos de diferentes disciplinas, con la identificación de problemas y la búsqueda de soluciones novedosas desde el aprendizaje de las Ciencias Naturales, con la elaboración de conclusiones sobre e estudio de procesos y fenómenos naturales, en hacer la valoración del resultado de su trabajo y del trabajo de los demás, en la determinación de los conocimientos que requieren ser tratados el perfeccionamiento de su modo de actuación en el proceso de enseñanza-aprendizaje. 
Por lo que existe la necesidad de resolver el siguiente problema científico: ¿Cómo contribuir al modo de actuación profesional pedagógico en la formación inicial de los profesores de Biología y Química en la Universidad de Pinar del Río "Hermanos Saíz Montes de Oca?

El objetivo de este trabajo es elaborar tareas docentes centradas en la determinación y solución de problemas didácticos, de forma tal que propicien el desarrollo del modo de actuación profesional pedagógico durante el proceso enseñanza-aprendizaje de la formación inicial de los profesores de Biología y Química en la Universidad de Pinar del Río "Hermanos Saíz Montes de Oca".

\section{La formación del modo de actuación profesional pedagógico}

Como parte del proceso de formación de los profesionales de la educación se incluye el proceso de formación del modo de actuación profesional pedagógico, mediante el cual se propicia el desarrollo del modo de actuación que se basa en el dominio pleno de los conocimientos, habilidades, valores y capacidades más generales relacionadas con el objeto de la profesión.

Teniendo en cuenta que el desarrollo del modo de actuación profesional pedagógico es una parte del proceso de formación del profesional de la educación se realiza un análisis sobre las definiciones que brindan diferentes autores respecto al modo de actuación profesional en el ámbito educativo.

El concepto modo de actuación profesional ha sido definido por diversos investigadores, entre ellos autores cubanos: García (1996); Álvarez (1999).

A partir de un estudio sobre las definiciones planteadas por los citados investigadores, las autoras de este trabajo consideran que el desarrollo de la creatividad humana es esencial para el desarrollo de un modo de actuación profesional, pero es necesario precisar cuáles son las acciones de la actividad generalizada que debe ejecutar el estudiante que se está formado como profesional en el logro de este modo de actuación.

Por otra parte Álvarez (1999) precisa que el modo de actuación del profesional debe evaluarse desde el proceso de formación y dar continuidad en el egresado. Así es asumido por las autoras del trabajo ya que el modo de actuación se va desarrollando desde la formación como profesional durante el transcurso de la carrera y que en ese proceso de formación profesional el estudiante va mostrando modos de actuación en cada una de las actividades en que se desempeña.

Además, para que el egresado pueda resolver problemas del objeto de su trabajo en el objeto de su profesión también depende del desarrollo de la creatividad humana, logrando una actuación competente comprometida consigo mismo y con la sociedad.

Se tiene en cuenta que el profesional de la educación, una vez formado, debe dirigir el proceso educativo para lograr la formación integral de las nuevas generaciones en la educación general media básica y media superior, lo que indica que el profesor formador de profesores, en la dirección del proceso de formación del profesional, debe contextualizar sistemáticamente la materia que se enseña a cada uno de los niveles educacionales que constituyen la esfera de actuación del profesional. 
De modo que así el estudiante aprende y aprende cómo se enseña esa materia. Y esos son modos de actuación profesional pedagógico que se materializan en la dirección del proceso de enseñanza-aprendizaje durante la formación del profesoral de la educación.

Diferentes investigadores cubanos han abordado la temática referente al modo de actuación profesional pedagógico en el ámbito educativo:Chirino (2002); Fuxá(2003); Pla(2005); Páez (2010),Addine(2013).

El estudio de los aportes sobre el modo de actuación profesional pedagógico dado por los autores ya citados permiten apreciar criterios comunes como: sistema y secuencia de acciones generalizadoras del docente. Estoscriterioscomunesson planteados porPla (2005) yAddine(2013).

Chirino (2002) y Parra (2007) consideran el modo de actuación profesional pedagógico como pensamento estratégico para aprender y educar con identidad profesional, actitud pedagógica y empleo de métodos de trabajo pedagógico.

La definición sobre el modo de actuación profesional pedagógico tratada porPáez (2010), precisa como elemento esencial el desarrollo de la interdisciplinariedad.

En la elaboración de la definición del concepto de modo de actuación profesional pedagógico se tuvo en cuenta también la brindada por Pla (2005), quien lo considera como:

La dirección de la dinámica en la que se produce la educación de los alumnos desde la institución escolar y que tiene entre sus componentes principales al docente y su influencia es decisiva en el logro de los objetivos formativos por los alumnos. (p. 16)

A partir de las definiciones dadas por los investigadores antes referidos, es definido por la autoras de este trabajo,el modo de actuación profesional pedagógico del profesor de Ciencias Naturales como: la manera de dirigir el proceso de enseñanza-aprendizaje como parte del proceso pedagógico a partir de una secuencia de acciones generalizadas que debe seguir el docente en su misión educativa; entre las acciones se distinguen el estudio y la búsqueda de nuevos conocimientos a partir de la integración de contenidos de diferente disciplinas y de los conocimientos que requieren ser tratados para la profundización, la identificación de problemas y sus soluciones novedosas desde la enseñanza de las Ciencias Naturales así como la elaboración de conclusiones sobre el estudio de procesos y fenómenos naturales con la aplicación de los conocimientos teóricos en la actividad práctica y la valoración del resultado de su trabajo y del trabajo de los demás. De manera que el profesional pueda identificar en una situación docente el objetivo y el motivo, el contenido, el método, los medios de enseñanza, presentar alternativas, avizorar y prever resultados, establecer modificaciones, determinar conocimientos necesarios para alcanzar objetivos, integrar conocimientos, habilidades, valores y sentimientos.

El profesor en su formación inicial se va apropio de su modo de actuación en la medida que logre desarrollar el interés por conocer lo nuevo, por buscar incesantemente y explorar sus ideas, las de otros, las del texto, contexto, sus vivencias, argumentos, 
experiencias, llegar a nuevas conclusiones, valorar paulatinamente acerca de sí mismo sobre el papel desempeñado.

Acciones que deben conducir a: la aplicación de los conocimientos teóricos en la actividad práctica, a la solución de tareas que impliquen la intradisciplinariedad y la interdisciplinariedad, a la elaboración de conclusiones a partir de las ideas esenciales de la clase, a la aplicación de conocimientos para la solución de tareas que expresan contradicciones, a la identificación de elementos del conocimiento que requieran ser tratados en el estudio independiente, a la autovaloración y valoración del trabajo de los demás en la solución de tareas, a la elaboración de respuestas novedosas en la solución de tareas.

\section{Los problemas didácticos en el proceso enseñanza-aprendizaje de la formación inicial del modo de actuación profesional pedagógico de los profesores de Biología}

Calero (2005) define los problemas didácticos como:

La expresión de contradicciones entre un estado actual y un estado deseado, la cual puede ser de diversos tipos, tanto en el plano cognoscitivo, como en el eminentemente práctico y se relaciona con la actuación del docente en la dirección del proceso de enseñanza y aprendizaje, es decir, en la ejecución de las acciones propias de la función docente - metodológica. (p. 40)

La definición dada porCalero (2005) ha sido enriquecida en este trabajo, por sus autoras, definiendo como problemas didácticos: la expresión de contradicciones entre un estado actual y un estado deseado, la cual puede ser de diversos tipos, tanto en el plano cognoscitivo, como en el eminentemente práctico y se relaciona con la actuación del docente en la dirección del proceso de enseñanza y aprendizaje en la formación inicial del modo de actuación profesional pedagógico de los profesores de Biología y Química.

Es consideración de las autoras del trabajo que describe que los problemas didácticos para resolverse en el proceso de enseñanza-aprendizaje, antes deben ser identificados, determinando las contradicciones que dificultan alcanzar el objetivo en el proceso de enseñanza-aprendizaje de la formación inicial del modo de actuación profesional pedagógico de los profesores de Biología-Química y que requieren ser aclaradas y resueltas de forma adecuada.

Estimular la determinación de problemas didácticos en los profesores en su formación inicial conduce a problematizar el conocimiento y la realidad. Las contradicciones pueden darse entre lo que conoce el profesor en formación y lo desconocido para él; entre lo que sabe y lo que necesita saber; entre lo que hace y lo que debería hacer; entre cómo lo hace y cómo debe hacerlo.

Según Rubinstein (1997):

El factor inicial del proceso mental es, por regla general, la situación problémica.

El hombre empieza a pensar cuando siente la necesidad de comprender algo. El pensar empieza normalmente con un problema o con una cuestión, con un asombro o con una confusión, con una contradicción. (p.85) 
El planteamiento de Rubinstein (1997) es asumido por las autoras al considerar que la presentación de la situación problémica en la dirección del proceso de enseñanzaaprendizaje hace que se inicie el proceso de búsqueda en la formulación del problema por parte de los estudiantes, teniendo en cuenta para la presentación de la situación problémica, las necesidades, los motivos y los intereses de los estudiantes, es decir, los aspectos emocionales que expresan las vivencias subjetivas del hombre, esto se corresponde con el aspecto subjetivo del problema, entendido como interiorización de una contradicción, para la que no se conocen las vías a emplear.

Es enfatizado por Calero (2005) que resolver problemas es elemental en la formación del profesor, lo que se evidencia al plantear que:

Los problemas, por sus resultados o vías de solución sorprendente, constituyen para el futuro profesor un fuerte incentivo en función de despertar el interés y la motivación al planificar, ejecutar, controlar y evaluar el proceso de enseñanza aprendizaje de sus estudiantes. Los esfuerzos cognoscitivos y la tensión intelectual contribuyen de forma eficiente al proceso de búsqueda activa de la solución, lo que se expresa en la necesidad de alcanzar un propósito, condición esencial para lograr que el acto de resolver el problema no se convierta en un hecho rutinario en su solución. (p. 42)

En la determinación y solución de problemas didácticos, el profesor en formación debe emplear procedimientos, los que, a consideración de las autoras, deben elevar los intereses profesionales y a la vez exigir profundizar en los conocimientos, habilidades, hábitos, sin tener un algoritmo o analogía prefijado para el problema en cuestión, desarrollando las potencialidades como futuro profesor y caracterizando su modo de actuación profesional pedagógico.

Por lo que se ha planteado sobre el valor didáctico y formativo que tiene resolver problemas, en la formación inicial del modo de actuación profesional pedagógico, en la dirección del proceso de enseñanza-aprendizaje del profesor en formación se hace necesario trabajar con la habilidad solución y planteamientos de problemas pues "es esencial si se desea desarrollar en los estudiantes un tipo de pensamiento reflexivo y creativo". (Zilberstein, 2002, p. 85)

Partiendo del análisis de las deficiencias manifestadas en el modo de actuación profesional pedagógico de los estudiantes de la carrera Biología-Química y el análisis realizado sobre la teoría de problemas didácticos, la autora considera, que son problemas didácticos a solucionar en el proceso de enseñanza-aprendizaje de la carrera los siguientes:

- El estudio y la búsqueda de nuevos conocimientos a partir de la integración de contenidos de las diferente disciplinas biológicas y químicas.

- La identificación de problemas y sus soluciones novedosas desde la enseñanza de las disciplinas biológica y química.

- La elaboración de conclusiones sobre el estudio de procesos y fenómenos naturales desde las disciplinas biológicas y químicas.

- La aplicación de los conocimientos teóricos de las disciplinas biológicas y químicas en la actividad práctica. 
- La determinación de los conocimientos de profundización, por parte de los estudiantes, que requieren ser tratados para erradicar deficiencias.

- La valoración crítica del resultado del trabajo por parte del estudiante y del trabajo de los demás.

Tareas docentes centradas en la determinación y solución de problemas didácticos para la formación inicial del modo de actuación profesional pedagógicas en la dirección del proceso de enseñanza-aprendizaje de los profesores de Biología-Química

Las autoras del trabajo, asumen la definición detarea docente brindada por Fuxá (2004), quien plantea:

Es una unidad organizativa del proceso que condiciona en su estructuración sistemática, las acciones de aprendizaje que permite que el estudiante al realizarlas pueda adquirir conocimientos, desarrolle habilidades y reflexione conscientemente sobre las diversas acciones que realiza en el proceso de solución de dichas tareas; esto permite regular su comportamiento como aprendiz y educador, la interacción con los demás sujetos que intervienen en el proceso de solución, al establecer determinadas pautas de comportamiento y relaciones sociales, que junto al contenido curricular potencian la formación de valores morales, estéticos e ideológicas que se correspondan con los objetivos del proceso enseñanza-aprendizaje.(p. 230)

A partir de esta definición de tarea docente dada por Fuxá (2004), las autoras del trabajo concibe en las tareas docentes un sistema de acciones para la búsqueda y exploración del conocimiento por el estudiante desde posiciones reflexivas y problematizadas de la teoría y la práctica pedagógica, orientando la reflexión y análisis considerando los roles de aprendiz y educador; en la determinación y solución de problemas didácticos en el proceso de enseñanza-aprendizaje de la formación inicial del modo de actuación profesional pedagógico de los profesores de Biología y Química.

Se tiene en cuenta a la hora de concebir la tarea docente que en ella se presenten los componentes del proceso de enseñanza-aprendizaje por lo que se asume lo brindado por Álvarez (1999) quien plantea:

En la tarea docente está presente un objetivo, condicionado por el nivel de los estudiantes, por sus motivaciones e intereses, por la satisfacción de cada uno de ellos en la ejecución de la tarea (...). Hay un contenido a asimilar, una habilidad a desarrollar. El método en la tarea docente, es el modo en que lleva a cabo cada estudiante la acción para apropiarse del contenido. Por medio de la evaluación se explicita si ejecutó correctamente la tarea (...).(p. 45)

Las tareas docentes que se elaboran para el modo de actuación profesional pedagógico centradas en la determinación y solución de problemas didácticos en la formación inicial de profesores de Biología y Química, conciben un sistema de acciones para la búsqueda y exploración del conocimiento por el estudiante desde posiciones reflexivas y problematizadas de la teoría y la práctica pedagógica con orientación hacia la reflexión y el análisis. 
Ejemplo de una tarea docente centrada en la determinación y solución de problemas didácticos para el desarrollo del modo de actuación profesional pedagógico

La tarea docente que se presenta corresponde a los contenidos de asignatura Botánica I de la disciplina Botánica.

Problema a resolver con la tarea docente: ¿Cómo contribuir al modo de actuación profesional pedagógico en la formación inicial de los profesores de Biología y Química, en la Universidad de Pinar del Río "Hermanos Saíz Montes de Oca", desde el estudio de la Morfofisiología de las Cormofitas de la Botánica I?

Objetivo general de la tarea docente: Contribuir al modo de actuación profesional pedagógico en la formación inicial de los profesores de Biología y Química, en la Universidad de Pinar del Río "Hermanos Saíz Montes de Oca".

Objetivo específico de la tarea docente. Explicar el proceso de absorción de agua de las plantas precisando las relaciones estructura-función y organismo-medio externo parteindo de las características anatómicas y de las propiedades físico-químicas de las sutancias.

Cuestionario.

¿Cómo es posible la absoción del agua por las plantas si esta ocurre en sentido contrario a la fuerza de gravedad?

¿Como favorece el funcionamiento de los estomas a la incorporación del agua a las estructuras especializadas de la raíz?

¿Qué comportamiento hídrico de las células del mesófilo puede favorecer la absorción de agua por la planta, siempre que el resto de los factores externos e internos de la planta sean favorables? Explique.

¿Cómo evidenciar la relación propriedades químicas de los vasos del xilema y del agua con el transporte que ocorre de esta sustancia en contra a la fuerza de gravedad?

Analice las siguientes situaciones y comparta el critério personal con los demás. Sobre las situaciones dadas, explique lo que puede suceder con la absorción de agua por la planta.

A-Suelo saturado al que se le añaden sales en exceso.

B-Se igualan las concentraciones hídricas medio externo de la raíz y las del jugo vacuolar del pelo absorbente.

Precisa las condiciones del médio externo de la planta en el clima tropical cubano que pueden favorecer la absorción de agua y caules otros factores considera que deban tenerse presente para hacer este análisis.

Diseña un experimento para demostrar el sentido del transporte de agua por la planta.

La tarea docente se realiza en clase práctica de la temática seleccionada, se realiza de forma independiente y será evaluada teniendo en cuenta el desarrollo de las habilidades en los estudiantes. El cuestionario que refiere la tarea docente presentada 
favorece el estudio y la búsqueda de nuevos conocimientos a partir de la integración de contenidos de la disciplina Botánica ya que en este caso se tratan propiedades de las sustancias que favorecen la función vegetativa sobre el transporte del agua. Se solucionan situaciones problémicas relacionadas con este contenido y los estudiantes trabajan en función de aportar sus soluciones novedosas, se presentan situaciones que los estudiantes deben analizar y elaborar conclusiones sobre el estudio del proceso de absorción de agua. En la solución de la tarea docente los estudiantes aplican conocimientos teóricos de la disciplina Botánica en la actividad práctica lo que les permite la determinación de los conocimientos de profundización, por parte de los estudiantes, que requieren ser tratados para erradicar deficiencias. La exposición de las respuestas permite realizar la valoración crítica del resultado del trabajo por parte del estudiante y del trabajo de los demás.

\section{Conclusiones}

El modo de actuación profesional pedagógico del profesor de Ciencias Naturales es la manera de dirigir el proceso de enseñanza-aprendizaje como parte del proceso pedagógico a partir de una secuencia de acciones generalizadas que debe seguir el docente en su misión educativa; entre las acciones se distinguen el estudio y la búsqueda de nuevos conocimientos a partir de la integración de contenidos de diferente disciplinas y de los conocimientos que requieren ser tratados para la profundización, la identificación de problemas y sus soluciones novedosas desde la enseñanza de las Ciencias Naturales así como la elaboración de conclusiones sobre el estudio de procesos y fenómenos naturales con la aplicación de los conocimientos teóricos en la actividad práctica y la valoración del resultado de su trabajo y del trabajo de los demás.

La secuencia de acciones generalizadas que debe seguir el docente en su misión educativa y que forman parte de la definición presentada en este trabajo como modo de actuación profesional pedagógico del profesor de Ciencias Naturales son asumidas como problemas didácticos a resolver desde la tarea docente.

Las tareas docentes que se elaboran para el modo de actuación profesional pedagógico centradas en la determinación y solución de problemas didácticos en la formación inicial de profesores de Biología y Química, conciben un sistema de acciones para la búsqueda y exploración del conocimiento por el estudiante desde posiciones reflexivas y problematizadas de la teoría y la práctica pedagógica con orientación hacia la reflexión y el análisis contribuyendo así al desarrollo del modo de actuación profesional pedagógico del profesor de Ciencias Naturales.

\section{Referencias}

Addine, Fernández, F. (2004).Didáctica, teoría y práctica. La Habana, Cuba: Editorial Pueblo y Educación.

Addine Fernández, F. (2013).La Didáctica General y su Enseñanza en la Educación Superior Pedagógica. Aportes e Impacto. La Habana, Cuba: Editorial Pueblo y Educación.

Álvarez de Zayas, C. M. (1999).Didáctica. La escuela en la vida. La Habana, Cuba: Editorial Pueblo y Educación. 
Calero Fernández, N. L. (2005). El modo de actuación creativo del profesor enformación(Tesis Doctoral). Instituto Superior Pedagógico“Félix Varela”. Villa Clara, Cuba.

Calzado Lahera, D. (2003). Un modelo de Forma de Organización de la Docencia en la formación inicial del profesor(Tesisdoctoral). Instituto Superior Pedagógico "Enrique José Varona". La Habana, Cuba.

García Ramis, L. (1997). Autoperfeccionamiento docente y creatividad. La Habana, Cuba: Editorial Pueblo y Educación, 67.

Chirino Ramos, M. V. (2002). Perfeccionamiento de la formación inicial investigativa de los futuros profesionales de la educación(Tesis doctoral). Instituto Superior Pedagógico "Enrique José Varona". La Habana, Cuba.

FuxáLavastida, M. (2004).Un modelo didáctico curricular para la auto preparación docente de los estudiantes de licenciatura en Educación Primaria (Tesis doctoral).Universidad de Ciencias Pedagógica "Rafael María de Mendive". Pinar del Río, Cuba.

Páez Rodríguez, B. (2010). Modelo pedagógico para la formación del modo de actuaciónprofesional pedagógico interdisciplinario, en los estudiantes en formación como docentes, para la Secundaria Básica de Pinar del Río. (Tesis doctoral). Universidad de Ciencias Pedagógicas "Rafael María de Mendive". Pinar del Río, Cuba.

Plá López, R. (2005). Fundamentos del enfoque integral y contextualizado actuación del docente. Modo de actuación del docente desde un enfoque integral y contextualizado (Tesis doctoral). Universidad de Ciencias Pedagógicas "Manuel AscunceDomenech". Ciego de Ávila, Cuba.

Rubinstein, J. L. (1997). Principios de la Psicología General. La Habana, Cuba:Editorial Pueblo y Educación.

ZilbersteinToruncha, J. (2002). Hacia una didáctica desarrolladora. La Habana,Cuba: Editorial Pueblo y Educación. 